\title{
Revista de Historia de la Psicología
}

www.revistahistoriapsicologia.es

\section{Besos peligrosos: José Ingenieros y la mirada del criminólogo}

\author{
Ana Elisa Ostrovsky \\ Universidad Nacional de Mar del Plata. CONICET.
}

Luis Alberto Moya

Universidad Nacional de Mar del Plata.

\section{N F O R M A C I Ó $\mathrm{N}$ A R T}

Recibido: 7 mayo 2020

Aceptado: 31 enero 2021

Palabras clave

beso,

delito,

José Ingenieros,

positivismo,

Argentina

\begin{abstract}
R E S U M E N
La recepción de la criminología italiana en Argentina, de la mano de autores como José Ingenieros, se ancló en el problema de la cuestión social del momento. El proceso modernizador de finales de siglo XIX y comienzos del XX, que supuso la secularización de sus instituciones y el fortalecimiento del modelo agroexportador, tuvo como contrapunto una inmigración masiva y una urbanización poco planificada que conllevaron un alto grado de conflictividad social. Entre los objetos abordados por la nueva ciencia se encontraba el alcoholismo, la prostitución, pero también pequeñas conductas inapropiadas. Uno de esos pequeños objetos plausibles de convertirse en delito fue el beso. El presente trabajo constituye un análisis de una presentación llamada El delito de besar, que José Ingenieros realizara sobre dicho tema. En el mismo, el autor efectúa una reconstrucción desde la mirada criminológica del acto de besar, instalando como criterio clasificatorio la pregunta sobre el consentimiento.
\end{abstract}

\section{Dangerous kisses: José Ingenieros and the criminologist's look}

\section{A B S T R A C T}

The reception of Italian criminology in Argentina, by authors such as José Ingenieros, was anchored in the social problem of the moment. The modernization process of the late nineteenth and early twentieth centuries, which involved the secularization of its institutions and the strengthening of the agro-export model, had as a counterpoint a massive immigration and unplanned urbanization that led to a high degree of social conflict. Among the objects addressed by the new science are alcoholism, prostitution, but also small inappropriate behaviors. One of those small plausible objects of become a crime was the kiss. The present work constitutes an analysis of a presentation called The crime of kissing, which José Ingenieros will make on this subject. In it, the author made a reconstruction from the criminological view of the act of kissing, installing as a classification criterion the question about consent.

\section{Introducción}

Argentina, desde finales de siglo XIX y comienzos del XX se organizó, al igual que los países vecinos de la región, como un Estado
Nación de corte moderno. Si bien dicho proceso no tuvo características homogéneas en todo el país, en la ciudad de Buenos Aires y en la Cuenca del Rio de La Plata, presentó un carácter compacto en la organización de sus instituciones. (Bohoslavsky, 2015). En el plano económico se

Correspondencia Ana Elisa Ostrovsky: anaelios@gmail.com Luis Alberto Moya: luigimoya@gmail.com

ISSN: 2445-0928 DOI: https://doi.org/10.5093/rhp2021a1

C 2020 Sociedad Española de Historia de la Psicología (SEHP) 
consolidaba el modelo de acumulación del capital agroexportador y en las elites gobernantes se profundizaba la preocupación por un proceso inmigratorio poco planificado y su deriva en una población conflictiva y heterogénea (Suriano, 2004). Cabe destacar, como un elemento clave de la ciencia social del momento, que el proceso inmigratorio se desarrolló fuera de las expectativas de los proyectos de los pensadores románticos y los intelectuales positivistas. Así, lejos de poblar el país en su extensión, el aluvión inmigratorio se concentró en los principales centros urbanos, particularmente en la ciudad de Buenos Aires, aumentando su población de 171000 habitantes en 1869 a 1563000 en 1914, dentro de los cuales el 49\% eran inmigrantes (Caimari, 2009). Dicha masividad se reflejaba en desocupación, anonimato, hacinamiento $\mathrm{y}$ falta de condiciones higiénicas, mostrando a medida que transcurría el nuevo siglo, un amento del delito en las estadísticas confeccionadas por la policía porteña y una considerable participación de inmigrantes en ellos, como así también en la población de los nacientes hospicios (Vezzetti, 1983).

El proceso modernizador citado, requería de herramientas y tecnologías capaces de comprender e intervenir socialmente, el positivismo como una verdadera filosofía en acción, se convirtió en una grilla interpretativa aplicable a diferentes esferas de la vida colectiva. Así nacen fecundos ámbitos de estudio de la identidad y los problemas de la nación, y florece la sociología, la criminología y el estudio del carácter vernáculo y su mentalidad (Lancelotti, 1912; Vilanova, 2001). El positivismo como modo naturalista y científico de explicación, posibilitaba conciliar el reformismo propuesto por la elite liberal a la vez que permitía sostener la tradición conservadora del gobierno vigente (Candioti, 2002; Talak y Miranda, 2010). Bajo su perspectiva se reformó el código penal, pasando de la teoría clásica de la pena centrada en el acto a una moderna concepción centrada en el sujeto, sus características y por lo tanto su potencial de peligrosidad (Salvatore, 2005). La mirada científica del delincuente se trasladó a las instituciones destinadas al mismo, produciéndose un movimiento de creación y reforma de instituciones estatales como el Depósito de Contraventores de la Policía de la Capital Federal (1899). El mismo estaba anexo a la cátedra de Medicina Legal de la Universidad de Buenos Aires y en su estructura contenía una Sala de Observación de Alienados bajo la responsabilidad de José Ingenieros (Constantin, 2018).

\section{José Ingenieros y la criminología}

José Ingenieros (1877 - 1925) en la primera década del siglo XX se convirtió en el principal referente de la criminología a nivel local a partir de una elaboración teórica que desplazaba el elemento antropomórfico en la etiología de los criminales, por una clasificación de corte psicopatológica que distinguía anomalías de tipo volitivo, intelectual y moral. Las mismas, que podían presentar un carácter tanto congénito como adquirido, estaban en la base explicativa de la comisión del delito y fueron explicadas de forma sistemática en su obra Criminología de 1910 (Sozzo, 2011). El autor fue un polifacético filósofo y médico de origen italiano radicado tempranamente en la Argentina que se destacó, en el campo de la psiquiatría, la criminología, la historia y la política constituyéndose además en un referente de las juventudes del momento. En el marco de las disciplinas de lo psíquico representó a la psicología naturalista de comienzos de siglo XX en Argentina como Jefe de Clínica Médica en la cátedra de José María Ramos Mejía, profesor de la cátedra de Psicología Experimental en la Universidad de Buenos Aires y fundador de la Sociedad de Psicología de Buenos Aires. Fue también el redactor de las primeras crónicas historiográficas sobre la psicología del momento (Ingenieros, 1919a). Escritor de célebres obras como La simulación de la locura (1903), Histeria y sugestión (1904), Principios de Psicología (1911), El hombre mediocre (1913) y La locura en Argentina (1919). En el plano editorial fue secretario de redacción de la revista La Semana Médica, colaborador de Criminología Moderna y director de los Archivos de Psiquiatría y Criminología. Como señalamos, el autor obtuvo la jefatura del Servicio de Observación de Alienados de la Policía Federal y del Instituto de Criminología de la Penitenciaría Nacional de Buenos Aires. En ese ámbito, su labor clínica tuvo como objetivo la prevención del crimen y la evaluación de la culpabilidad de los delincuentes, con todos los efectos legales y penales que esto podía conllevar (Fernández, 2009).

El desarrollo de la criminología del último cuarto del siglo XIX presenta como figura ineludible al médico italiano Cesare Lombroso, que con su trabajo Ĺuomo delinquente (1876) articuló teorías evolucionistas con mediciones antropométricas efectuando mediciones de cráneos de delincuentes con el objetivo de encontrar patrones comunes (Galeano, 2013). Dicha empresa lo llevó a postular la existencia de una vinculación entre rasgos biológicos endógenos propios de la constitución física del sujeto y la tendencia innata a delinquir. Su línea de pensamiento, llamada escuela antropológica o criminología positivista italiana impactó fuertemente en el desarrollo de la criminología mundial propiciando una matriz de explicación naturalista del delito y una posibilidad científica de prevención (Dunnage, 2018). A nivel local, en 1898, había sido publicada la revista Criminología Moderna, fundada por Pietro Gori, un médico italiano representante de las ideas lombrosianas y en 1902 se fundaba la revista Archivos de Psiquiatría y Criminología Aplicadas a las Ciencias Afines que jugaría un papel clave en el proceso de consolidación a nivel nacional y continental de la disciplina (Mailhe, 2014). En dicho contexto, José Ingenieros, atravesado por la influencia de César Lombroso, Rafael Garófalo, y Enrico Ferri, representantes de la escuela criminológica italiana, presentó tempranamente un marcado interés por los problemas criminológicos y psiquiátricos de su época, focalizándose en el estudio del sujeto criminal y su relación con el derecho penal de la época. En efecto, para la legislación entonces vigente, determinar la locura en los criminales era razón suficiente para su excarcelación, ya que no cumplía con el requisito de intencionalidad requerido por la ley penal para declararlo culpable (Levaggi, 2016). La propia Gina Lombroso, criminóloga e hija del afamado médico italiano, visitó la Argentina en 1907 comentándole a su padre la buena impresión que le había causado la tarea de Ingenieros: "Si hubieran querido extraerte de la cabeza todo lo que has deseado hacer en favor de los delincuentes, a fin de ponerlo en práctica, no se habría podido realizar nada mejor de lo que aquí he visto". (como se citó en Ruíz Díaz, 2016). Sin embargo, como vimos precedentemente, en contraposición a las ideas lombrosianas, el autor no ubicaba las causas del delito en determinantes meramente morfológicos del individuo. Cuestionando la tesis lombrosiana del delincuente nato, su esquema de análisis 
incluía un maridaje entre factores endógenos y exógenos, emergiendo como variable de análisis la dinámica psíquica.

\section{El delito de besar}

El delito de besar, escrito de 1902 por Ingenieros, si bien tiene el formato de conferencia, es un ensayo que integra su obra $L a$ Psicopatología en el Arte, aunque algunas ediciones posteriores lo individualizaron como tal. En el mismo, observamos un entrecruzamiento de sus estudios de derecho, medicina y las notas del periodo que nos convoca. Hay análisis criminológico, reflexión sociológica, estudio legal, crítica social, referencias literarias y también un dejo de tono burlón en algunos pasajes que se erigen como indudablemente humorísticos. El interjuego entre el lenguaje poético, las precisiones del derecho penal y las referencias médicas muestran un estilo peculiar donde arte y ciencia, literatura y casuística se encuentran recíprocamente enriquecidos (Fernández, 2012).

Para ubicarnos en el contexto de producción del texto, cabe señalar que lo produce un José Ingenieros joven, que está comenzando su producción científica sin por ello deslindarse de sus apetencias literarias. Al comenzar el siglo pasado aún se encontraba pivoteando entre su militancia socialista, que lo impulsa a fundar el periódico La Montaña y sus burlonas aventuras modernistas con el grupo la Syringa, cofradía de bohemios destinaba a jugar bromas a las formas rígidas de la intelectualidad de su tiempo. En efecto, quienes se han encargado de analizar la obra del autor como Torchia Estrada (1967) dividen su producción científica en cuatro periodos: el socialista (hasta 1900), el psicológico (entre 1900 - 1911), el filosófico (1911 a 1918) y el historiador de la cultura, que alcanza hasta el año de su fallecimiento en 1925.

En ese momento, con veinticinco años, se estaba forjando como psiquiatra realizando sus prácticas en el Servicio de Observación de Alienados del Depósito de Contraventores a cargo de su ex profesor y amigo Francisco de Veyga. Como observamos, la escuela positivista italiana y eventos como la visita del criminólogo Pietro Gori al país lo habrían impactado, siendo los problemas criminológicos y la pregunta por la simulación una piedra angular de sus obras en el campo de intersección entre la psiquiatría y la ley (Ingenieros, 1904, 1916, 1919).

En su análisis del beso, el autor se adentra en dicho objeto ubicándolo como un problema jurídico. La pregunta que guía su argumentación es bajo qué circunstancias un beso puede considerarse un acto delictuoso. Para comenzar a responderla en un segundo apartado desarrolla la distinción entre dos tipos de beso: el beso tierno y el beso erótico o "beso casto" y "beso de amor". El beso casto es aquél dado entre parientes o amigos, que caracteriza a las despedidas y los encuentros, propios de las costumbres domésticas o ceremoniales de cada latitud con un límite de tolerancia variable. El parentesco aparece como un dato relevante, también la edad del besador, como menciona socarronamente las prebendas del anciano que habiéndose apagado sus fervores puede besar a doncellas.

El beso de amor consentido o recíproco se produce entre personas que tienen responsabilidad de sus actos y no es delito, aunque puede variar en intensidad y calidad, desde lo platónico hasta la lujuria, desde lo casto hasta la voluptuosidad (Ingenieros, 1902).

\section{El beso ilícito}

El carácter delictivo de un beso está entonces en su intención y en el status del consentimiento de quien lo recibe. Al respecto, para el autor, las costumbres eróticas y los pasos del cortejo complejizarían la tarea del besador, el cual vale destacar es siempre pensando como varón y por supuesto en clave heterosexual como lo indicaba el carácter conservador de la ciencia del momento. Ingenieros se adentra así en el consentimiento, un tema complejo que en nuestros días aparece como un núcleo discursivo de debates feministas. El autor afirma que desde el punto de vista psicológico resulta imposible que el agente pueda tener verdadera certidumbre del consentimiento, sobre todo a raíz de la existencia de un pudor femenino puesto en juego en nombre de la virtud. Por eso:

Es simple cuestión de tacto y de prudencia el saber en qué momento el corazón pide que sí mientras las palabras dicen que no ...(...)...El que pretende besar un minuto antes, se expone a violentas resistencias; el que se decide a besar un minuto después, merece que lo desprecien. (Ingenieros, 1902, p.5).

Así la tarea del varón residiría en ser un verdadero termómetro de los complejos grados de consentimiento, tema siempre difícil en tanto en dichos años, que una mujer manifestara su voluntad deseante, la colocaba bajo la sospecha social de la deshonestidad sexual, de ser una mujer fácil, insana o ambas (Barrancos, 2002)

Siguiendo con su voluntad taxonómica, el autor sostenía que el beso no consentido podía jurídicamente clasificarse entre las “injurias de hecho". En ese sentido, citando el Código Penal Argentino refería: "Comete el delito de injuria, el que deshonra, desacredita o menosprecia a otro por medio de hechos o acciones que no importen otro delito de más gravedad" (art. 179). Las injurias son graves cuando "tales acciones sean tenidas en concepto público por afrentosas en razón de su naturaleza, ocasión y circunstancias" (art. 180); si no median esas condiciones las injurias son reputadas leves (art. 181). (Ingenieros, 1902, p.5). Por lo tanto, desde su punto de vista, aunque el consentimiento siempre sea un tema difícil de establecer, la ausencia del mismo desata un acto injuriante hacia la persona besada.

\section{¿Ella se lo buscó?}

Una de las circunstancias agravantes del beso como delito es su publicidad. En ese sentido, el autor señala que no es usual el hecho de que una mujer querelle por un beso recibido estando a solas con el besador. El carácter privado del mismo desprotege a la mujer, porque el simple hecho de estar a solas con un varón es per se sospechosa. En este punto el autor refiere "Se presume que si ha estado a solas con él mediaba un principio de consentimiento tácito, o por lo menos una imprudencia que ha podido favorecer el error del agente" (Ingenieros, 1902, p.6). Así, en la presunta víctima reside la responsabilidad del acto. De alguna manera, la mujer que se encuentra a solas con varón se expone o al menos no hace nada por evitar el ilícito en ciernes. Pese a dicha interpretación, solidaria a la visión conservadora del varón como impulsivo sexual y a la mujer como encargada de su propia defensa, 
Ingenieros también menciona que otro modo de enfocar el asunto no es concentrarse en el pudor defensivo y la ofensa de la persona que recibe el beso, sino en la intención erótica de quien lo da.

En este punto el psiquiatra exhibe su temprano interés psicopatológico de entender al delito apoyándose en la criminología italiana centrada en el sujeto al abordar la poderosa noción de peligrosidad y enfocarse en las intenciones del besador. La Teoría Clásica se centraría, como observamos, en el acto mismo (el beso indeseado) más allá del agente. De esta forma, lo característico del delito no radicaría en la "injuria de hecho" privada, sino la ofensa a la honestidad implicada por la intención erótica del agente, intención burda, patológica, maliciosa. Hay entonces en el autor un balance en colocar al delito del besar entre la "injuria de hecho", cuando el agente tiene malicia erótica en su intención y es penado por ello y el "atentado al pudor", cuando más allá de las intenciones lo sancionado es el daño en la reputación de la víctima y su pudor defensivo. Se trata, en una escala leve, del núcleo duro de los debates criminológicos de la época en donde se penan las consecuencias de los actos o las intencionalidades de los agentes, ambos muy difíciles de separar y discernir en circunstancias con fronteras tan porosas y difusas. Con todo, un eje que toma el nivel criminólogo es el de la premeditación y la alevosía como agravantes, por excluir ambas el problema del consentimiento de la víctima. Para ejemplificar el asunto Ingenieros recurre al caso del estudiante ruso Ivanoff, deportado a Siberia por haber dado un "beso de amor" en circunstancias que sólo le autorizaban a dar "un beso de amistad" en un periplo de lo más escandaloso que incluye beso robado, sustracción de vehículo para darse a la fuga y tiroteo policial posterior.

Otro punto que toma el autor y que muestra la codificación y análisis de comportamientos cotidianos por parte de la criminología, es la interacción del consentimiento de los amantes con las costumbres de cada país, que obviamente condicionan la publicidad de esta prueba de amor. Ingenieros menciona así ciudades "cultas" donde hay tolerancia a las manifestaciones públicas de amor $\mathrm{y}$ ciudades como Buenos Aires donde incluso el mismo hecho en otra latitud permitido daba lugar a una intervención policial por escándalo. Aquí se introduce una variante cultural y epocal que le pone ciertos límites al afán propiamente naturalista del investigador: Buenos Aires quiere parecerse a Paris, pero no es Paris... Pese a ello, hay algo instintivo presente y la hermosura de la mujer pareciera ser siempre animalmente una fuerza que despierta una biología instintiva y por lo tanto irrefrenable en su complementaria contraparte masculina. En palabras del autor "Justa advertencia para los desposados que realizan su viaje de bodas, máxime cuando la belleza de la esposa favorezca el despertar de los afectos y deseos a que se refiere la discreta sentencia" (Ingenieros, 1902, p.8)

\section{Cuando lo que prima es la imprudencia}

Aun siendo consentido y privado, el autor se interroga sobre el beso que por su intensidad y tenacidad puede afectar la integridad física 0 provocar, si se repite, una debilitación general de la salud. En ambos casos la figura legal que se esgrime es la de delito de lesiones. Haciendo gala de su cultura literaria y científica el autor cita a médicos que se encargaron de dar criterios funcionales y anatómicos de tipos de besos, besos cutáneos, cutáneo-mucosos, entre mucosas, etc. Asimismo cita versos de poetas como Ronsard y Gabriel D’Annuunzio que señalan en sus exaltaciones literarias el carácter románticamente nocivo del beso apasionado. En estos casos extremos, esos besos que lastiman son para la psiquiatría en ciernes una muestra de franca patología mental bajo la forma del masoquismo. Se trata de manifestaciones anormales que se pueden encontrar en estados neurasténicos o directamente en la alienación mental, en palabras del propio autor. Otra posible veta de daño es la vía bucal como promotora de contagios y los irrisorios recaudos de los higienistas como el caso del galeno estadounidense Herman Sommer, inventor de un aparato con una pequeña pantalla de gasa antiséptica destinado a filtrar los besos. Ingenieros nuevamente con humor nos refiere que "El uso de este ingenioso invento yanqui no se ha difundido; la higiene es clarividente, pero el amor es ciego" (Ingenieros, 1902, p.11)

\section{A modo de reflexión}

Ingenieros en su curioso escrito nos muestra a modo de cierre cinco circunstancias claves para determinar el carácter delictuoso de un beso. A saber:

$1^{\circ}$ La coacción, por falta de consentimiento, que implica el delito de injuria.

$2^{2}$ La intención erótica, esencial para que exista el atentado al pudor

3ํㅡ La premeditación y la alevosía, agravantes de los delitos precedentes.

$4^{\circ}$ La publicidad que implica una contravención a las buenas costumbres, aunque no media coacción.

$5^{\underline{0}}$ La imprudencia, que por los efectos del besar puede constituir el delito de lesiones.

En cuanto a la reparación, en algunos casos es posible y en otros no. El dinero no repara la moral herida y tampoco se puede extender a toda una sociedad ofendida por el atentado a sus buenas costumbres. Con su estilo burlón, estilo que pareciera echar por tierra todo el afán clasificatorio exhibido en las líneas precedentes, el autor culmina su escrito esgrimiendo lo siguiente:

Para ser justos, podría autorizarse a la ofendida a devolver el beso que no ha deseado recibir, imponiendo al besador la obligación de soportarlo. Pues, al fin y al cabo, un beso suele ser un homenaje más bien que una ofensa, por lo menos en la intención de quien lo da; y si quien lo recibe tiene la ingratitud de no creerlo (Ingenieros, 1902, p.12).

A los efectos del presente artículo consideramos que revisar un escrito marginal en la vasta obra de José Ingenieros, un ensayo tal vez poco conocido del autor, nos sirve de pretexto para mostrar tres núcleos temáticos de diverso status epistemológico que pueden iluminar trabajos subsiguientes. El primero de ellos es el análisis del estilo literario del autor, la amalgama entre ciencia y literatura que sostuvo a lo largo de su obra y el reiterado recurso al humor. Al analizar el Delito de Besar nos podemos realizar los siguientes interrogantes ¿quién escribe?, ¿el taxónomo positivista, el poeta naturalista o el estudiante travieso? 
Otro núcleo de problemas que nos abre el análisis del texto radica en el estudio de los límites de la criminología moderna y la tipificación de comportamientos cotidianos alejados de los grandes cuadros delictivos, el papel de la nueva disciplina que en clave médico-jurídica intervenía en lo cotidiano de las personas, convirtiendo cuestiones otrora morales o religiosas en objetos de la ciencia natural y plausibles de ser analizados e investigados. Finalmente, un gran campo de problemas se abre en el estudio de los valores sexistas que tiñen todo el despliegue del análisis, la presunción de la necesaria reticencia de la mujer honesta, el si por el no, la actividad masculina y la pretendida pasividad femenina en donde aparece el ejemplo de la mujer que buscaría el beso porque se cita a solas con el malintencionado besador, entre otras. Con todo, sin caer en visiones presentistas del texto, si bien el feminismo en Argentina se escribía al calor de los primeros años del siglo XX, nos llama la atención su actualidad al poner el foco de análisis y el estudio del posible delito en comportamientos comúnmente naturalizados en el discurrir cotidiano. El beso robado nos lleva al problema del consentimiento, mostrándonos preocupaciones que están lejos de las simples simpatías con lo antiguo y nos colocan en la vigorosa agenda del feminismo contemporáneo.

\section{Referencias}

Barrancos, D. (2002). Inclusión/Exclusión. Historia con Mujeres [Inclusion/ Exclusion. History with Women]. FCE.

Bohoslavsky, E. (2015). El rostro humano de la historia del Estado [The human face of the history of the State]. Estudios Sociales del Estado, 1(1), 239-247. https://doi.org/10.35305/ese.v1i1.16

Caimari, L. (2004). Apenas un delincuente. Crimen, cultura y castigo en la Argentina [Hardly a criminal. Crime, culture and punishment in Argentina]. Siglo XXI.

Candiotti, M. (2002). Comentario a Zimmermann: Los liberales reformistas. La cuestión social en Argentina 1890-1916 [Comment to Zimmermann: The Liberal Reformists. The social question in Argentina 1890-1916]. Delito $y$ Sociedad, 1(17), 163-165. https://doi.org/10.14409/dys.v1i17.5435.

Constantin, S. T. (2018). Sugestión, delito y multitud: positivismo criminológico y nacimiento del Estado Nación (El caso de" La mal vida en Buenos Aires") [Suggestion, crime and multitude: criminological positivism and the birth of the Nation State (The case of "The bad life in Buenos Aires")]. Alegatos, 19(61)591-604.

Dunnage, J. (2018). The work of Cesare Lombroso and its reception: Further contexts and perspectives. Crime, Histoire $\mathcal{E}$ Sociétés/Crime, History $\mathcal{E}$ Societies, 22(2), 5-8. https://doi.org/10.4000/chs.2258

Fernández, C. (2009). Las historias de vida en José Ingenieros [The life stories in José Ingenieros]. Anclajes, 13(13), 71-89.

Fernández, C. (2012). José Ingenieros y los saberes modernos [José Ingenieros and modern knowledge]. Alción.

Galeano, D. (2013). Civilización y delito: Notas sobre cuatro criminólogos argentinos [Civilization and Crime: Notes on Four Argentine Criminologists]. Revista de historia del derecho, (45), 265- 277.

Ingenieros, J. (1949). Simulación de la locura [Insanity simulation]. Primera edición, 1903 Roggero.
Ingenieros, J. (1957) El delito de besar [The crime of kissing]. En La psicopatología en el arte [sychopathology in Art]. Primera edición 1902. Elmer Editor.

Ingenieros, J. (1962). Histeria y sugestión [Hysteria and suggestion]. Obras completas. Tomo II (pp. 7-166). Primera edición, 1904. Mar Océano.

Ingenieros, J. (1916): Principios de psicología [Principles of psychology]. Buenos Aires: Rosso.

Ingenieros, J. (1913). El hombre mediocre [The mediocre man]. Renacimiento.

Ingenieros, J. (1962). Criminología [Criminology]. Obras completas. Tomo II. (pp. 259-474). Primera edición, 1916. Mar Océano..

Ingenieros, J. (2011). Los estudios psicológicos en la Argentina [Psychological studies in Argentina]. En Antología de textos clásicos de la psiquiatría latinoamericana (pp. 33-51). Primera edición, 1919. CULagos Ediciones.

Ingenieros, J. (1962). La locura en la Argentina [Madness in Argentina]. Obras completas. Tomo II. (pp. 167-258). Primera edición, 1919. Mar Océano.

Lancelotti, A. (1912). La criminalidad en Buenos Aires: 1885 a 1910 al margen de la estadística [Crime in Buenos Aires: 1885 to 1910 on the margins of statistics]. Revista argentina de ciencias políticas, 4, 326-342.

Levaggi, A. (2016). El derecho penal argentino en la historia [Argentine criminal law in history]. Eudeba.

Mailhe, A. (2014). El archivo de Archivos. Un latinoamericanismo eurocéntrico en la psiquiatría y la criminología de principios del siglo XX [The Archives archive. A Eurocentric Latin Americanism in early 20th century psychiatry and criminology]. Varia historia, 30(54), 655-678. https://doi.org/10.1590/ S0104-87752014000300004

Ruíz Díaz, M. (2016). Europa y America: Guglielmo Ferrero y Gina Lombroso en la Penitenciaría Nacional de Buenos Aires (1907) [Europe and America: Guglielmo Ferrero and Gina Lombroso in the National Penitentiary of Buenos Aires (1907)]. Anales del IAA, 46. 77-90.

Salvatore, R. (2005). Sobre el surgimiento del estado médico legal en la Argentina (1890-1940) [On the emergence of the legal medical status in Argentina (1890-1940)]. Estudios Sociales, 20(1), 81-114. https://doi.org/10.14409/ es.v20i1.2464

Sozzo, M. (2011). Los exóticos del crimen. Inmigración, delito y criminología positivista en la Argentina (1887-1914) [The exotics of crime. Immigration, crime and positivist criminology in Argentina (1887-1914)]. Delito $y$ Sociedad, 20(32), 19-51. https://doi.org/10.14409/dys.v2i32.5647

Suriano, J. (2004). La cuestión social en Argentina, 1870-1943 [The social question in Argentina, 1870-1943]. Buenos Aires: Agencia Nacional de Promoción Científica y Tecnológica, Ministerio de Cultura y Educación, Secretaría de Ciencia y Técnica.

Talak, A y Miranda, M. (2010). Progreso, degeneración y darwinismo en la primera psicología argentina, 1900-1920. En Derivas de Darwin: cultura y política en clave biológica [Progress, degeneration and Darwinism in the first Argentine psychology, 1900-1920. In Darwin's Drifts: culture and politics in a biological key]. pp. 299-320. Buenos Aires: Siglo XXI.

Torchia-Estrada, J. C. (1967). Tres pensadores en la vida intelectual argentina: Ingenieros, Korn, Romero [Three thinkers in Argentine intellectual life: Ingenieros, Korn, Romero]. Journal of interamericana Studies, 9(1), 248-272.

Vezzetti, H. (1983). La locura en la Argentina [Madness in Argentina]. Folios Ediciones.

Vilanova, A. (2001). El carácter argentino: Los primeros diagnósticos [The Argentine character: The first diagnoses]. Universidad Nacional de Mar del Plata. 